\title{
Privacy matters
}

\section{Welchen Wert hat der Schutz persönlicher Daten bei Online-Girokonten?}

\author{
Julian Kettl • Klaus Schäfer • Niklas Buchauer
}

Eingegangen: 19. April 2020 / Angenommen: 22. April 2021 / Online publiziert: 27. Mai 2021

(C) Der/die Autor(en) 2021

Zusammenfassung Finanztransaktionen werden zunehmend online abgewickelt, generell scheinen FinTechs das traditionelle Bankgeschäft zu verdrängen. Damit stellt sich vermehrt die Frage, inwiefern der Schutz personenbezogener Daten bei Online-Finanzdienstleistungen in der Wahrnehmung des Kunden als Differenzierungsmerkmal gesehen werden kann. Welchen Wert haben diese Daten aus Sicht der Bankkunden? Lassen sie sich durch Finanzdienstleister monetarisieren? Der vorliegende Artikel zeigt mithilfe der Präferenzmessmethode Choice-Based-Conjoint-Analyse, dass Studierende im Allgemeinen großen Wert auf den Schutz ihrer Privatsphäre legen und bereit sind, eine deutlich höhere monatliche Grundgebühr zu akzeptieren, wenn ihre Daten ausreichend von dem Zugriff Dritter geschützt sind. Schon wenige, besonders relevante Eigenschaften eines Girokontos (v. a. Datenschutz \& Privatheit sowie Grundgebühr) tragen dazu bei, dass sich Studierende für ein bestimmtes Girokonto entscheiden. Der Wert, den Studierende der Privatsphäre beimessen, hängt dabei von verschiedenen Faktoren, wie der Vorerfahrung mit Online-Finanzdienstleistungen oder dem Geschlecht der jeweiligen Person, ab.

Schlüsselwörter Privatheit · Persönliche Daten · Choice-Based Conjoint-Analyse · FinTech · Online-Finanzdienstleitungen

\footnotetext{
Julian Kettl $(\bowtie) \cdot$ Klaus Schäfer $\cdot$ Niklas Buchauer Lehrstuhl für Betriebswirtschaftslehre I: Finanzwirtschaft und Bankbetriebslehre, Universität Bayreuth, 95440 Bayreuth, Deutschland

E-Mail: julian.kettl@uni-bayreuth.de

Klaus Schäfer

E-Mail: klaus.schaefer@uni-bayreuth.de

Niklas Buchauer

E-Mail: Niklas.buchauer@uni-bayreuth.de
} 


\title{
Privacy Matters
}

What is the Value of Protecting Personal Data in Online Checking Accounts?

\begin{abstract}
Financial transactions are increasingly being processed online, and in general, FinTechs seem to be displacing traditional banking. This raises the question of the extent to which the protection of personal data in online financial services can be seen as a differentiating feature in the perception of the customer. What is the value of this data from the bank customers' point of view? Can financial service providers monetize them? Using the preference measurement method choice-based conjoint analysis, this article shows that students generally attach great importance to the protection of their privacy and are willing to accept a significantly higher monthly fee if their data is sufficiently protected from third-party access. Just a few, particularly relevant characteristics of a current account (especially data protection \& privacy as well as basic fee) contribute to the fact that students decide on a specific current account. The value students attach to privacy depends on various factors, such as previous experience with online financial services or the gender of the person concerned.
\end{abstract}

Keywords Information privacy - Personal data C Choice-based conjoint analysis · Fintech · Online financial services

\section{Relevanz und Motivation}

Durch das Aufkommen moderner Informationstechnologien können zunehmend mehr personenbezogene Daten ${ }^{1}$ gesammelt, aufbewahrt und analysiert werden. Aufgrund des überlegenen Werbeeffekts personalisierter Werbung rücken diese Daten auch bei Finanzdienstleistern in den Fokus ökonomischer Entscheidungen und die Anzahl von Unternehmen steigt, deren Geschäftsmodell auf dem Sammeln, Verarbeiten, Kaufen und Verkaufen personenbezogener Daten basiert.

Die große Mehrheit der Bevölkerung sieht die Preisgabe personenbezogener Daten als zum modernen Leben dazugehörig an. Gleichzeitig gehen begründete Bedenken damit einher, dass digital gespeicherte Personendaten leicht über das Internet verbreitet werden können und es unklar ist, wer zu welchem Zweck Zugang zu diesen Daten hat (Dinev und Hart 2006a). Diese Bedenken werden im Folgenden als Privatheitsbedenken bezeichnet. Privatheit ist vom geläufigen Begriff der Privatsphäre abzugrenzen. Privatsphäre ist jener nichtöffentliche Bereich, in dem sich ein Mensch ungestört von äußeren Einflüssen entfalten kann. Unter Privatheit dagegen versteht man die Möglichkeiten einer Person, selbst zu entscheiden, wie sie sich darstellt und wie viel sie von sich preisgeben möchte. Einige Forscher definieren Privatheit als physische Privatheit und sprechen vom ,right of the individual to be let alone" (Warren und Brandeis 1890), während philosophische Ansätze Privatheit als ,a state or condition of limited access to a person“ (Schoeman 1984) definie-

\footnotetext{
1 Persönliche bzw. personenbezogene Daten sind Daten, mit Hilfe derer man Personen differenzieren und voneinander abgrenzen kann (Jentzsch et al. 2012).
} 
ren, in dem der Grad der Privatheit dadurch bestimmt ist, in welchem Ausmaß Dritte Zugang zu personenbezogenen Informationen anderer haben. Werden diese Privatheitsbedenken ${ }^{2}$ von im Internet agierenden Unternehmen berücksichtigt, kann dies unter bestimmten Umständen für die Unternehmen als auch für Kunden mit einem Mehrwert einhergehen. So zeigen Studien, dass Dienstleister einen Preisaufschlag verlangen können, wenn sie für den Schutz personenbezogener Daten bei OnlineDienstleistungen sorgen. Hann et al. (2007) stellen fest, dass Studierende erhebliche Privatheitsbedenken bei der Nutzung von Online-Dienstleistungen haben. Sie zeigen, dass stärker auf Online-Dienstleistungen zugegriffen wird, wenn eine monetäre Entlohnung angeboten wird. Zugleich sind Kunden bereit, Preisaufschläge zu akzeptieren, wenn Datenschutzerklärungen leichter zugänglich gemacht und prominenter platziert werden (Tsai et al. 2011). Privatheitsbedenken können reduziert werden, wenn Personen vor Abschluss einer Transaktion über die unternehmensinternen Praktiken der Datenverwendung aufgeklärt werden (Culnan und Armstrong 1999). Eine Untersuchung der Unternehmensberatung PwC Strategy (2020) zeigt, dass vor allem Vorbehalte gegenüber dem Datenschutz die Verbreitung von Open Banking in Europa hemmen. So sind nur $20 \%$ der Umfrageteilnehmer aus zwölf europäischen Ländern bereit, persönliche Finanzdaten mit Banken oder Drittanbietern zu teilen, 55\% lehnen dies ab. Demgegenüber sind es häufig Online-Konten, die ein kostenloses Girokonto ermöglichen. Die Stiftung Warentest erklärt in ihrem Magazin „Finanztest“, dass gerade während der Corona-Pandemie kostenlose OnlineKonten interessant werden können, da sich Personen, die bisher eher bar bezahlt haben, nun mit möglichen Gebühren bei Zahlungen mit der Girokarte konfrontiert sehen (FAZ 2020). In einer Verbraucherumfrage zum Thema Digital Banking durch die Unternehmensberatung EY, gab knapp die Hälfte der Befragten an, dass Sicherheitsbedenken ein Hinderungsgrund für die Nutzung von Apps oder Onlineangeboten von Finanzdienstleistern sind. Außerdem vertrauen die Verbraucher dem Bankberater vor Ort deutlich öfter als einer Onlinebank (EY 2021).

Für Unternehmen ist es wichtig zu wissen, welchen Wert Kunden dem Schutz ihrer persönlichen Daten zuweisen, denn so können sie sich für bestimmte Datenschutzmaßnahmen entscheiden und eine bessere Marktpositionierung erreichen (Acquisti et al. 2013). Über den genauen Wert personenbezogener Daten herrscht jedoch eine kontroverse Diskussion. Einige Forscher sehen einen besonderen Schutz personenbezogener Daten als Wettbewerbsvorteil an (Tsai et al. 2011). Bei gleichen Preisen für eine bestimmte Dienstleistung kann gezeigt werden, dass Anbieter, welche mehr Wert auf den Schutz personenbezogener Daten legen, höhere Marktanteile erzielen können, dieser Vorteil allerdings durch Preisunterbietungen schnell aufgehoben wird (Jentzsch et al. 2012). Tsai et al. (2011) kommen zu dem Ergebnis, dass Individuen bei Onlinekäufen auf den günstigsten Preis achten, allerdings dann bereit sind, einen Preisaufschlag zu zahlen, wenn ihnen aktiv aufgezeigt wird, welchen Da-

\footnotetext{
2 In der vorliegenden Arbeit wird aufgrund der fortschreitenden Digitalisierung und Online-Datenverarbeitung das Konzept der informationellen Privatheit zugrunde gelegt. Dieses beschäftigt sich mit den personenbezogenen Daten, welche gesammelt, verarbeitet und analysiert werden können (Bélanger und Crossler 2011). Diese Möglichkeiten führen dazu, dass Privatheit als ökonomisches Gut, welches im Austausch gegen andere Waren und Dienstleistungen gehandelt werden kann, fungiert (Smith et al. 2011).
} 
tenschutzbestimmungen sie unterliegen. Eine mittels Choice-Based-Conjoint (CBC) Analyse durchgeführte Studie, welche die Relevanz verschiedener Merkmale für die Entscheidungsfindung bei einem App-Kauf durchführt, kommt sogar zu dem Ergebnis, dass Studierende bei der Wahl der App dem Schutz ihrer Privatheit einen höheren Stellenwert zuweisen als dem Preis (Buck et al. 2017). Huberman et al. (2005) können ebenfalls zeigen, dass Personen für die Preisgabe bestimmter personenbezogener Daten einen sehr hohen Preis verlangen, dieser allerdings kontextabhängig ist und mit dem Grad der Sensibilität der Daten variiert. So bewerten Individuen Daten höher, wenn diese mit ihren Finanzen in Verbindung stehen, als wenn sie rein demographisch sind. Des Weiteren wird der Wert abhängig vom Datenempfänger unterschiedlich wahrgenommen (Carrascal et al. 2013; Huberman et al. 2005).

Auch die Bereitschaft zur Preisgabe personenbezogener Daten ist kontextabhängig und hängt nicht zwangsläufig vom objektiven Level des Datenschutzes ab, sondern geht mit dessen relativer Veränderung zu einem dem Käufer bekannten Referenzlevel einher (Adjerid et al. 2018). Personen neigen ebenso dazu, für den Schutz ihrer Daten weniger bezahlen zu wollen, als sie für die Preisgabe ihrer Daten erstattet bekommen möchten (Acquisti et al. 2013).

Im Gegensatz zu diesen Ergebnissen stehen Studien, die den Schutz der Privatheit nicht als werthaltig bemessen. So finden beispielsweise Beresford et al. (2012) heraus, dass minimale Preisunterbietungen unabhängig von den geforderten Daten bei der Transaktion dazu führen, dass die kostengünstigeren Anbieter gewählt werden.

Individuen treffen ihre Transaktionsentscheidungen online anhand diverser Faktoren und wägen $\mathrm{ab}$, ob ein bestimmtes $\mathrm{Ma}$ an Privatheit aufgegeben wird, um Vorteile aus der Datenpreisgabe zu ziehen (Dinev und Hart 2006a). Um herauszufinden, welche Eigenschaften Individuen bei ihren Entscheidungen als relevant erachten, wird im Bereich der Finanzdienstleistungen oft auf die Conjoint-Analyse zurückgegriffen, da Finanzdienstleistungen eine Vielzahl von Eigenschaften mit jeweils verschiedenen Ausprägungen besitzen (Zinkhan und Zinkhan 1990). So bedienen sich Rolfes et al. (2005) einer Limit Conjoint-Analyse, um die optimale Konfiguration eines Girokontos für Studierende zu ermitteln und kommen zu dem Ergebnis, dass vor allem die Kontoführungsgebühr ein wichtiges Entscheidungskriterium ist. Speziell auf Online-Finanzdienstleistungen zielt eine Studie von Verma et al. (2004) ab. Mittels CBC finden die Autoren heraus, dass die Transaktionsgebühren entscheidend für die Wahl eines Online Brokers sind. Beide Studien beziehen allerdings den Umgang mit personenbezogenen Daten als Entscheidungskriterium nicht mit ein. Gerade im Bereich von Finanzdienstleistungen könnte dies allerdings ein wichtiges Entscheidungskriterium sein, da hier besonders sensible Daten gesammelt werden (Huberman et al. 2005) und Girokonten zunehmend gebührenpflichtig sind. Durch eine wirtschaftliche Verwertung personenbezogener Daten könnten onlinebasierte Direktbanken und neue digital getriebene Finanzdienstleister (FinTechs) die Kontoführungsgebühren senken und sich so einen Wettbewerbsvorteil sichern.

Der vorliegende Artikel behandelt die Frage, welchen Wert Kunden ihren personenbezogenen Daten beim Abschluss von Online-Finanzdienstleistungen beimessen. Es wird die Bedeutung von Privatheit und des Umgangs mit persönlichen Daten bei der Wahl des Girokontos bei Studierenden unter Zuhilfenahme einer CBC untersucht. Insbesondere wird dabei aufgezeigt, welche Relevanz dem Entscheidungskri- 
terium Privatheit im Vergleich zu anderen Entscheidungskriterien beigemessen wird, um im Ergebnis den Wert personenbezogener Daten abschätzen zu können. In der angewandten CBC werden Studierende als Probanden herangezogen, da diese ein hohes erwartetes zukünftiges Einkommen besitzen und deren Bedürfnisbefriedigung für Kontoanbieter als besonders wichtig anzusehen ist (Kara et al. 1994; Rolfes et al. 2005).

\section{Privatheit und personenbezogene Daten bei Finanzdienstleitungen}

Finanzdienstleistungen werden zunehmend online abgewickelt. Schon 2014 haben zwei Drittel der Deutschen mindestens einmal wöchentlich Online-Banking genutzt (EY 2014). In einer Befragung des Bundesverbands Informationswirtschaft, Telekommunikation und neue Medien e. V. (Bitkom) gaben $22 \%$ der Internetnutzer an, dass sie Finanzgeschäfte über FinTechs abwickeln würden (Bitkom 2014). Während die Zahl an Bankfilialen seit Jahren rückläufig ist, stieg beispielsweise von 2009 bis 2017 die Anzahl der Online-Girokonten um 58\% auf 67 Mio. (Deutsche Bundesbank 2019). Die Corona-Pandemie hat diesen Trend weiter verstärkt.

Das Volumen der potenziell adressierbaren Märkte für FinTechs aus den Bereichen Finanzierung und Vermögensverwaltung beträgt in Deutschland knapp 1,7 Billionen $€$. Dorfleitner et al. (2016) prognostizieren für diese Bereiche, welche 2015 ein Marktvolumen von 2 Mrd. $€$ aufwiesen, ein starkes Wachstum auf etwa $148 \mathrm{Mrd}$. $€$ Marktvolumen im Jahr 2035. Um Schwierigkeiten in der Abgrenzung zwischen FinTechs und Direktbanken zu vermeiden, wird in der dieser Arbeit zugrundeliegenden Befragung die Nutzung von Direktbanken mit der Erläuterung versehen, dass es sich dabei um Banken ohne eigenes Filialnetz handelt.

Im Januar 2016 trat die zweite Zahlungsdiensterichtlinie (Payment Service Directive 2, PSD2) der Europäischen Kommission in Kraft, welche seit Januar 2018 auch in Deutschland in nationales Recht umgesetzt ist. Die PSD2 soll insbesondere die Sicherheit und den Verbraucherschutz bei Zahlungsdiensten und Zahlungsdienstleistern erhöhen. Durch die sog. OpenAccess-Regelung, welche Drittanbietern nun nach expliziter Erlaubnis der Kontoinhaber den Zugriff auf deren Kontodaten ermöglicht, könnten herkömmliche Kreditinstitute einen Wettbewerbsvorteil gegenüber FinTechs verlieren, da diese nun ebenfalls Kontoinformationen der Kontoinhaber weiterverarbeiten und so passende Dienstleistungen anbieten können (Dorfleitner et al. 2016). Darüber hinaus müssen sich Verbraucher beim Einkaufen im Internet nicht mehr in das Online-Banking ihres Kreditinstituts einloggen, um Bezahlvorgänge abzuschließen. Bezahlvorgänge können direkt von Zahlungsdienstleistern getätigt werden. Ebenfalls können alternative Finanzdienstleister wie bspw. Kontoinformationsdienste auf alle Konten der Verbraucher zugreifen und den Verbrauchern ihre Kontostände und Umsätze so in aufbereiteter Form anzeigen. Außerdem können Sie algorithmisch erkennen, welche Verträge, zum Beispiel für Strom oder Telefonie, der Kunde zu welchen Kosten abgeschlossen hat und bieten ihm automatisiert kostengünstigere Verträge von Drittanbietern an. Schließt der Kunde solche Verträge $\mathrm{ab}$, erhält der Kontoinformationsdienst eine Provision des Drittanbieters. 


\section{Ermittlung entscheidungsrelevanter Kriterien bei der Wahl eines Girokontos}

\subsection{Methodik und Aufbau der Untersuchung}

Um die Bedeutung der Privatheit bei der Wahl von Onlinekonten zu ermitteln, wurde für die vorliegende Untersuchung die Präferenzmessmethode Conjoint Analyse (CA) angewandt. Dabei bewerten die Probanden nicht einzelne Eigenschaften von Produkten, sondern ihnen wird eine Kombination aus verschiedenen Eigenschaften mit dazugehörigen Ausprägungen, sog. Stimuli, vorgelegt. Eine Variante der CA ist die in dieser Arbeit angewandte CBC, bei der die Teilnehmer in sogenannten Choice-Tasks jeweils wählen, für welche der ihnen vorgelegten Stimuli sie sich entscheiden oder ob sie sich für keinen der Stimuli entscheiden (sog. None-Option) (Balderjahn et al. 2009; Cohen 1997). Dabei wird zuerst der Gesamtnutzen einer Produktalternative ermittelt und dann in Nutzenbeiträge für einzelne Eigenschaften heruntergebrochen (Baier und Brusch 2009). Dieses Verfahren hat bei der Präferenzmessung den Vorteil, dass es aufgrund seiner Realitätsnähe zu genaueren Ergebnissen führt, als wenn jede Eigenschaft eines Produkts oder einer Dienstleistung einzeln bewertet und diese dann zu einem Gesamtnutzen zusammengefügt werden würden (Baier und Brusch 2009). Außerdem gleichen die einzelnen Choice-Tasks reellen Kaufentscheidungsprozessen (Cohen 1997).

Die hier durchgeführte CBC-Analyse ist in einen Fragebogen eingebettet, um sowohl demographische als auch weitere für die Forschungsfrage relevante Merkmale abzufragen (Hann et al. 2007). ${ }^{3}$ An der Umfrage nahmen ausschließlich Studierende deutscher Universitäten teil, die finale Auswertung basiert auf 178 Rückmeldungen.

\subsection{Auswahl der Eigenschaften und Eigenschaftsausprägungen}

Die Ergebnisverwertung im Sinne einer konkreten Ableitung von Maßnahmen hängt in hohem Maße von der Auswahl der richtigen Eigenschaften ab. Um Eigenschaften sinnvoll zu wählen, müssen sie bestimmte Kriterien erfüllen (Weiber und Mühlhaus 2009). Aus Anwendersicht dürfen nur solche Eigenschaften abgefragt werden, welche für den Anwender auch tatsächlich umsetzbar sind. Außerdem müssen sie unabhängig, vollständig, beeinflussbar und realisierbar sein (Weiber und Mühlhaus 2009).

Das Kriterium der Beeinflussbarkeit wird in der vorliegenden CBC-Analyse zugunsten präziserer Forschungsergebnisse verletzt. Die Eigenschaft Datenschutz \& Privatheit ist nur bedingt durch Zahlungsdienstleister beeinflussbar. Deren erlaubte Ausprägungen hängen maßgeblich vom Gesetzgeber ab. Allerdings wird hier aus Forschungszwecken angenommen, dass FinTechs diese Eigenschaft aktiv beeinflussen können, um zu ökonomischen Einschätzungen über den Wert personenbezogener Daten zu gelangen.

Abb. 1 liefert eine Übersicht über die gewählten Eigenschaften und die jeweils drei Ausprägungen pro Eigenschaft (symmetrisches Design).

\footnotetext{
3 Der gesamte Fragebogen kann bei den Autoren kostenfrei per E-Mail angefordert werden.
} 


\begin{tabular}{|c|c|c|c|}
\hline Eigenschaften & \multicolumn{3}{|c|}{ Ausprägungen } \\
\hline Grundgebühr p. M. & $0 €$ & $5 €$ & $10 €$ \\
\hline Mögl. Geld abheben & nur an Automaten & nur in Supermärkten & Automaten \& Supermärkte \\
\hline Kosten Geld abheben & kostenfrei & $2 \times$ p.M. frei, dann $3 \%$ & $4 \times$ p.M. frei, dann $3 \%$ \\
\hline Kosten Überweisung & kostenfrei & $0,10 €$ & $0,20 €$ \\
\hline Datenschutz \& Privatheit & Standard mit Privatsphäre & Standard ohne Privatsphäre & Weitergabe Daten \\
\hline Zusatzleistungen & Girokarte & Girokarte, MasterCard & Giro, Master, Reisevers. \\
\hline
\end{tabular}

Abb. 1 Eigenschaften und Ausprägungen der Choice-Based-Conjoint-Analyse

Die Eigenschaften und deren Ausprägungen werden durch eine Dokumentenanalyse ermittelt. Im Rahmen dieser Dokumentenanalyse werden wissenschaftliche Aufsätze, die angrenzende Forschungsfragen behandeln (u. a. Buck et al. 2017; Zinkhan und Zinkhan 1990; Rolfes et al. 2005; Verma et al. 2004) sowie die aktuellen Konditionen von Direktbanken und FinTechs herangezogen. Die für die Untersuchung besonders relevante Eigenschaft Datenschutz und Privatheit wird in die Ausprägungen Standard mit Privatsphäre, Standard ohne Privatsphäre und Weitergabe Daten unterteilt. Standard mit Privatsphäre meint dabei, dass nur die für den Kontoerstellungsprozess benötigten Daten erhoben werden, ohne dass die kontoführende Bank diese Daten für weitere (Werbe-)Zwecke nutzt. Demgegenüber nutzt ein Finanzdienstleister bei der Ausprägung Standard ohne Privatsphäre Transaktionsda-

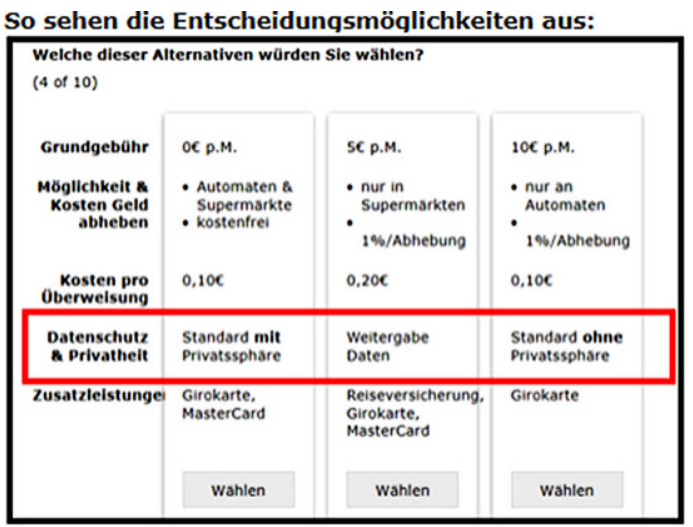

Bedeutung der einzelnen Ausprägungen bei Datenschutz \& Privatheit:

- Standard mit Privatsphäre : Bei Kontoerstellung werden nur Standarddaten wie Adresse und Geburtsdatum abgefragt, durch eine Datenschutzbestimmung erklärt der Anbieter, dass er keine sonstigen Daten speichert und für eigene Zwecke auswertet.

- Standard ohne Privatsphäre : Wie "Standard mit Privatsphäre", Anbieter darf aber Transaktionsdaten (Überweisungen, Abhebungen...) zu eigenen Zwecken speichern, verarbeiten und auswerten, um Ihnen bspw. eigene Finanzprodukte vorzuschlagen.

- Weitergabe Daten : Wie "Standard ohne Privatsphäre", zusätzlich darf Anbieter alle Transaktionsdaten an Dritte weitergeben.

Abb. 2 Erläuterung der Eigenschaft „Datenschutz und Privatheit“ 
ten des Kontoinhabers zu eigenen Zwecken, um dem jeweiligen Kunden passgenaue zusätzliche Angebote zu senden. Die Ausprägung Weitergabe Daten besagt, dass der Finanzdienstleister die Transaktionsdaten des Kontoinhabers an Dritte weitergeben darf, damit diese dann beispielsweise ihre Produkte individuell bewerben können. Die Durchsicht der Datenschutzbestimmungen ausgewählter FinTechs ergibt, dass diese weitestgehend der Ausprägung Standard ohne Privatsphäre der Eigenschaft Datenschutz \& Privatheit (Abb. 2) entsprechen.

Dabei ist zu beachten, dass noch weitere hier nicht abgefragte Faktoren bei der Wahl des Kontos bzw. des Kontoanbieters relevant sind, die aufgrund der Fokussierung auf die Privatsphäre hier nicht weiter beleuchtet wurden. So liegt nahe, dass Studierende eher bei Banken Konten unterhalten, bei denen bereits ihre Eltern ein Bankkonto haben oder bei denen sie bereits ein Schülerkonto hatten. Dieser Faktor ist in der vorliegenden Untersuchung deshalb nicht relevant, da die Kontomodelle realistische, aber fiktive Ausprägungen aufweisen und keinem existierenden Finanzdienstleister zugeordnet werden können. Auch die Rolle von Verbrauchertests und weitere Dienstleistungen, die nicht direkt in Verbindung mit dem Girokonto stehen, wie etwa eine persönliche Bankberatung, wurden nicht abgefragt. Zum einen verlieren manche dieser Dienstleistungen gerade bei jüngeren Kunden ohnehin an Bedeutung (Berger und Gensler 2007), zum anderen liegt der Fokus der Untersuchung auf der Auswahl verschiedener Kontomodelle. Das Untersuchungsdesign schließt zudem nicht aus, dass alle vorgestellten Kontomodelle einer einzigen Bank zuzuordnen sind, bei welcher der Kunde zwischen verschiedenen Modellen wählen kann. Die Zielsetzung der Untersuchung ist es, den Wert des Datenschutzes in das Verhältnis zur Grundgebühr zu setzen. Aus diesem Grund werden nur wenige weitere Faktoren (Möglichkeiten Geld abzuheben, Überweisungskosten, Zusatzleistungen) abgefragt, um einerseits einen Priming-Effekt zu vermeiden, ohne die Probanden andererseits mit zu vielen Ausprägungen zu überfordern. Während der Zusammenhang zwischen der individuellen Kundenerfahrung und dem Verbleib beim aktuellen Finanzdienstleister oder einem möglichen Wechsel bereits ausgiebig wissenschaftlich untersucht wurde, besteht hinsichtlich der Bedeutung externer Konsumententests und Kundenbewertungen noch weiterer Forschungsbedarf. Klar ist jedoch, dass Kunden heutzutage empfänglicher für Wettbewerber sind und trotz allgemeiner Zufriedenheit mit dem bisherigen Finanzdienstleister schneller und einfacher die Angebote der Konkurrenz erfassen und zu diesen wechseln können (Hansen und Malz 2015). Während Sicherheitsbedenken gerade bei Onlineanwendungen stets präsent sind, stellen Sicherheitsmechanismen bei Finanzdienstleistungen seit der Umsetzung der PSD2 und den damit einhergehenden einheitlichen Sicherheitsstandards für Finanzdienstleister kein entscheidendes Unterscheidungsmerkmal mehr dar.

\section{Ergebnisse der Untersuchung}

$31,5 \%$ der Studierenden geben an, dass sie bereits mindestens ein Girokonto bei einer Direktbank unterhalten. 24 nutzen dieses als ihr Hauptkonto, 32 als Zweitkonto und 14 Studierende geben sogar an, dass sie ausschließlich bei einer Direktbank ein Girokonto haben. Im Hinblick auf soziale Medien geben 93,3\% der Studierenden 
an, dass sie mindestens ein soziales Netzwerk nutzen. $12 \%$ der Studierenden, die soziale Medien nutzen, waren bereits einmal einem Datenmissbrauch auf sozialen Medien ausgesetzt. Rund 10\% aller 178 Studierenden waren mit Datenmissbrauch in finanzieller Hinsicht konfrontiert, zwei dieser Studierenden wurden sowohl Opfer von finanziellem Missbrauch als auch von Missbrauch im Hinblick auf soziale Medien. Der Datenmissbrauch auf sozialen Medien zeigte sich am häufigsten darin, dass der Account gehackt wurde (7\%), gefolgt von der Versendung von Spamnachrichten durch den eigenen Account (4\%). Unrechtmäßige Abbuchungen von der Kreditkarte $(6 \%)$ oder vom Konto $(4 \%)$ waren die meistgenannten Datenmissbrauchsfälle in finanzieller Hinsicht.

Ein erstes Anzeichen über die Relevanz der einzelnen Eigenschaften eines Girokontos liefern die relativen Häufigkeiten der gewählten Eigenschaftsausprägungen. Es zeigt sich, dass sowohl der Datenschutz als auch die Grundgebühr eine hohe Relevanz bei der Wahl eines Girokontos besitzen. Einerseits wurden Stimuli häufig gewählt, wenn sie keine Grundgebühr aufwiesen (34\%) oder wenn sie die höchste Datenschutzbestimmung hatten (42\%), andererseits sind die prozentualen Unterschiede der einzelnen Ausprägungen bei den Eigenschaften Grundgebühr sowie Datenschutz \& Privatheit hoch. Dies deutet darauf hin, dass sich die Studierenden bei ihrer Entscheidung stark auf die Ausprägungen dieser Eigenschaften konzentriert haben. Der Eigenschaft Zusatzleistungen hingegen kommt ein geringerer Stellenwert bei der Entscheidung zu. Treten in einem Stimulus die Eigenschaftsausprägungen $0 €$ p. M. sowie Standard mit Privatsphäre gemeinsam auf, wird dieser in $64 \%$ der auftretenden Fälle auch gewählt. Ein Chi-Quadrat-Test zeigt, dass sich die Ausprägungen innerhalb jeder Eigenschaft $(p<0,01)$ signifikant voneinander unterscheiden, und weist auf einen starken Interaktionseffekt der Eigenschaften Grundgebühr $\times$ Datenschutz \& Privatheit $(\chi 2=11,728 ; p<0,05)$ hin.

Mithilfe eines hierarchischen Bayes Modells kann gezeigt werden, dass Studierende dem Datenschutz mit 33,0\% Relevanz ( $\sigma=14,4 \%$; $\mathrm{KI}_{95} \%[30,86 ; 35,09]$ ) noch vor der Grundgebühr mit 24,9\% ( $\sigma=14,5 \%$; $\left.\mathrm{KI}_{95} \%[22,77 ; 27,04]\right)$ den höchsten Stellenwert bei der Wahl eines Girokontos zuweisen. Der Relevanzwert der Eigenschaft Datenschutz \& Privatheit ist damit signifikant höher als der Relevanzwert der Eigenschaft Grundgebühr. Weiterhin zeigen die Ergebnisse, dass der Stellenwert der Privatsphäre im Internet unabhängig vom Grad der Privatheitsbedenken der Studierenden ist. Auch die etwaige Nutzung sozialer Netzwerke im Hinblick auf den Stellenwert der Privatsphäre stellt bei der Wahl eines Girokontos kein Unterscheidungskriterium dar. Frauen weisen der Privatsphäre mit 35,2\% jedoch einen signifikant höheren Stellenwert zu als Männer mit 28,0\% $(p<0,01)$. Männern hingegen scheint die Grundgebühr mit 27,4\% bei der Entscheidung signifikant wichtiger zu sein als Frauen mit 21,9\% (T=2,39; $p<0,05)$.

Studierende mit positiven/neutralen Erfahrungen im Bereich der Online-Finanzdienstleistungen (Gruppe 1) legen bei der Wahl ihres Girokontos einen signifikant niedrigeren Stellenwert $(p<0,01)$ auf ihre Privatsphäre als diejenigen Studierenden, die noch keine Erfahrung mit Online-Finanzdienstleistungen gemacht haben (Vergleichsgruppe). Studierende, welche schon einmal Opfer von Datenmissbrauch bei Online-Finanzdienstleistungen (Gruppe 2) waren, weisen der Privatsphäre erstaunlicherweise ebenfalls einen signifikant niedrigeren Stellenwert $(p<0,01)$ als 
Tab. 1 Nutzenwerte HBModell

\begin{tabular}{ll}
\hline Eigenschaftsausprägung & $\begin{array}{l}\varnothing-\text { Nutzenwerte } \\
\text { (zero-centered) }\end{array}$ \\
\hline Grundgebühr & $-14,57$ \\
Nur an Automaten & 12,93 \\
Nur in Supermärkten & $-35,65$ \\
Automaten \& Supermärkte & 22,72 \\
Kostenfrei & 30,63 \\
2×p.M. frei, dann 3\%/ & $-27,34$ \\
Abhebung & \\
4×p.M. frei, dann 3\%/ & $-3,28$ \\
Abhebung & \\
Kostenfreie Überweisung & 32,95 \\
0,10€/Überweisung & $-1,04$ \\
0,20€/Überweisung & $-31,92$ \\
Standard mit & 103,98 \\
Standard ohne & $-18,66$ \\
Weitergabe & $-85,32$ \\
Giro & $-19,16$ \\
GiroMaster & $-1,16$ \\
ReiseGiroMaster & 20,33 \\
NONE & 86,61 \\
\hline &
\end{tabular}

die Vergleichsgruppe zu. Die Unterschiede zwischen den Gruppen 1 und 2 sind jedoch nicht signifikant ( $p$-Wert: 0,859). Die Ergebnisse deuten darüber hinaus darauf hin, dass Studierende, die weniger Wert auf ihre Privatsphäre legen, eher Online-Finanzdienstleistungen in Anspruch nehmen. Selbst negative Erfahrungen mit OnlineFinanzdienstleistungen führen bei dieser speziellen Gruppe nicht zu einem veränderten Verhalten, so dass keine Anpassung bei der Wertschätzung der Privatsphäre identifiziert werden kann. Der Stellenwert der Privatsphäre bei Studierenden, die Kunden einer Direktbank sind, ist signifikant niedriger als bei denjenigen, die kein Girokonto bei einer Direktbank unterhalten.

Nimmt man eine für CBC-Analysen gängige linear-additive Nutzenfunktion an, so kann der Wert der Eigenschaftsausprägung Weitergabe Daten durch den Nutzenvergleich der Eigenschaften Datenschutz \& Privatheit sowie Grundgebühr approximiert werden. Tab. 1 liefert einen Überblick über die Nutzenwerte der einzelnen Eigenschaftsausprägungen. Der Eigenschaft Grundgebühr liegt ein lineares Vektormodell zugrunde (Bichler und Trommsdorff 2009), ihr Nutzen kann daher wie folgt interpretiert werden: Eine Erhöhung der Grundgebühr um einen Euro geht mit einer Nutzenverringerung von 14,57 einher. Man erkennt, dass die Eigenschaftsausprägung Standard mit Privatsphäre den höchsten zusätzlichen Nutzen $(103,98)$ schafft, während die Ausprägung Weitergabe den Nutzen im Vergleich zu allen anderen Eigenschaftsausprägungen am stärksten reduziert $(-85,32)$.

Die 178 Studierenden würden dem Finanzinstitut im Durchschnitt für 4,58€= $(85,32-18,66) / 14,57$ der Weitergabe ihrer personenbezogenen Daten an Dritte - im Vergleich zum aktuell üblichen Fall einer Eigennutzung nur durch die Bank - zu- 
stimmen. Dabei bewerten Frauen die Weitergabe ihrer personenbezogenen Daten mit 5,33€ höher als Männer mit 3,80€. Kunden von Direktbanken messen der Weitergabe ihrer Daten einen Wert von $3,48 €$ bei, Kunden anderer Banken 5,20€.

\section{Implikationen für Wissenschaft und Praxis}

Es wird deutlich, dass Studierende der Wahrung der Privatsphäre auch im Bereich von Online-Finanzdienstleistungen einen hohen Stellenwert beimessen. Trotz allgemein bestehender Privatheitsbedenken kann gezeigt werden, dass Individuen in bestimmten Fällen zu einer Preisgabe personenbezogener Daten bereit sind und diese gemäß Privatheitskalkül oft rational begründet werden kann. Unternehmen können preisgegebene personenbezogene Daten vor allem mit Hilfe personalisierter Werbung monetarisieren. Diese Art von Werbung ist herkömmlicher Werbung in vielen Fällen überlegen.

Die Ergebnisse zeigen, dass die Bedeutung der Privatsphäre unabhängig davon ist, ob und in welchem Maße bei Studierenden Privatheitsbedenken vorliegen und ob diese positive, neutrale oder negative Erfahrungen mit Online-Finanzdienstleistungen gemacht haben. Allerdings treten Unterschiede in der Wertschätzung personenbezogener Daten auf. Direktbanken könnten diese Segmentierung zu ihrem Vorteil nutzen, in dem sie verschiedene Girokontomodelle anbieten, welche zwischen der Verwertung personenbezogener Daten und den direkten Kosten (bspw. Grundgebühr) abwägen. Bauer et al. (2019) sprechen der Preispsychologie, neben dem möglichst direkten Vertragsabschluss, einen deutlich positiven Effekt auf die Abschlusswahrscheinlichkeit und das Up- und Cross-Selling bei Finanzdienstleistern $\mathrm{zu}$.

Da Datenschutzbestimmungen einen hohen Stellenwert bei der Wahl eines Girokontos einnehmen, dienen sie girokontoanbietenden Finanzdienstleistern als besonderes Differenzierungsmerkmal und könnten dazu beitragen, auch weiterhin kostengünstige, ggf. sogar pseudo-kostenlose Girokonten anzubieten. Erkenntnisse aus der Untersuchung weisen darauf hin, dass Unternehmen für sie vorteilhaftere Datenschutzbestimmungen, die mit der Preisgabe und der Möglichkeit der Weiterverarbeitung einer großen Anzahl an Daten einhergehen, legitimieren können, wenn sie ein hohes Maß an Vertrauen schaffen. Hierfür sollten Finanzdienstleister ihre Datenschutzbestimmungen aktiv aufzeigen und ihre Kunden vor Vertragsabschluss mit diesen vertraut machen. Idealerweise schaffen es Finanzdienstleister, ihre Datenschutzbemühungen proaktiv und glaubwürdig aufzuzeigen. Informationen werden vermehrt online gesucht, Preise können leichter miteinander verglichen werden, auch soziale Netzwerke und Bewerbungsportale senken die Informationshürden. Marktteilnehmern können sich also einen Wettbewerbsvorsprung erarbeiten. Gleichzeitig eröffnet sich für klassische Banken die Chance, ihre jahrelang auf Sicherheit ausgelegte IT werbewirksam zu nutzen und sich so von Onlinefinanzdienstleistern abzugrenzen, die deutlich häufiger durch Datenschutzprobleme auffallen.

Vorangegangene Forschungsarbeiten zeigen, dass es für effektive personalisierte Werbung nicht zwangsläufig aller, sondern lediglich bestimmter personenbezogener Daten bedarf. Finanzdienstleister sollten sich, soweit möglich, auf die Aufzeichnung 
dieser Daten beschränken. Im Kontoerstellungsprozess haben zumindest FinTechs diesen Aspekt aber bereits erkannt, wie eine aktuelle Studie der Managementberatung Cofinpro (2021) zeigt. Bei der Eröffnung von Girokonten fragen Filialbanken deutlich mehr Datenfelder ab (bis zu 37), was mit einer erhöhten Abbruchquote quittiert wird. Die in der Studie Challengerbanken genannten FinTechs erheben deutlich weniger Daten (10-22 Datenfelder), um die Gewinnung von Neukunden stärker zu forcieren. Dieser vereinfachte Prozess der Kontoeröffnung geht aber gleichzeitig mit dem erhöhten Risiko für Sicherheitslücken einher, sodass Personen mit gefälschter Identität Konten eröffnen und für illegale Aktivitäten nutzen können.

Aus Sicht von Individuen zeigt sich, dass diese durchaus zu einer Preisgabe ihrer (Transaktions-)Daten bereit sind. Vor dem Hintergrund der aktuellen Gesetzgebung (DSGVO u. a.) und der damit einhergehenden geringeren Werbewirkung ist allerdings fraglich, ob es Direktbanken gelingen kann, mit diesen Daten einen höheren Mehrwert - als den Preisnachlass, den sie ihren Kunden für die Bereitstellung der Daten gewähren - zu generieren. Wie und in welchem Maße Finanzdienstleister durch personalisierte Werbung und andere auf personenbezogene Daten basierende Geschäftsmodelle Geld verdienen können, ist zum aktuellen Zeitpunkt nicht hinreichend erforscht und sollte, ebenso wie die Übertragung der vorliegenden Befragung auf weitere Personengruppen, einen Inhalt künftiger Forschung darstellen. Somit können die diskutierten Konsequenzen für Banken und FinTechs auf eine breitere Basis gestellt werden. Sicherlich werden die zur Verfügung stehenden Daten zumindest intern mittels Data Analytics zunehmend besser analysiert, um die für das eigene Geschäftsmodell relevanten Daten zu extrahieren. So können an das Kundenprofil maßgeschneiderte Angebote erstellt werden. Mit Hilfe der Datenanalyse identifiziert man darüber hinaus typische Gewohnheiten des Kontoinhabers und kann ihn im Falle einer Abweichung von erkannten Mustern vor eventuellen Betrugsfällen (z.B. APP-Fraud) warnen. Gleichzeitig ist es wichtig, die Kunden transparent über die Verwendung der Daten zu informieren und ihnen die persönlichen Vorteile sowie die Einhaltung der Datenschutzvorgaben vor Augen zu führen. Unternehmen können so durch eine geeignete Nutzung von Daten als dem „Öl der Zukunft“ (so Häcker und Bekelaer 2019) den Unternehmenswert nachdrücklich steigern.

Funding Open Access funding enabled and organized by Projekt DEAL.

Open Access Dieser Artikel wird unter der Creative Commons Namensnennung 4.0 International Lizenz veröffentlicht, welche die Nutzung, Vervielfältigung, Bearbeitung, Verbreitung und Wiedergabe in jeglichem Medium und Format erlaubt, sofern Sie den/die ursprünglichen Autor(en) und die Quelle ordnungsgemäß nennen, einen Link zur Creative Commons Lizenz beifügen und angeben, ob Änderungen vorgenommen wurden.

Die in diesem Artikel enthaltenen Bilder und sonstiges Drittmaterial unterliegen ebenfalls der genannten Creative Commons Lizenz, sofern sich aus der Abbildungslegende nichts anderes ergibt. Sofern das betreffende Material nicht unter der genannten Creative Commons Lizenz steht und die betreffende Handlung nicht nach gesetzlichen Vorschriften erlaubt ist, ist für die oben aufgeführten Weiterverwendungen des Materials die Einwilligung des jeweiligen Rechteinhabers einzuholen.

Weitere Details zur Lizenz entnehmen Sie bitte der Lizenzinformation auf http://creativecommons.org/ licenses/by/4.0/deed.de. 


\section{Literatur}

\section{Verwendete Literatur}

Acquisti A, John LK, Loewenstein G (2013) What is privacy worth? J Leg Stud 42(2):249-274

Adjerid I, Peer E, Acquisti A (2018) Beyond the privacy paradox —objective versus relative risk in privacy decision making. MISQ 42(2):465-488

Allenby GM, Ginter JL (1995) Using extremes to design products and segment markets. J Mark Res 32(4):392-403

Baier D, Brusch M (2009) Erfassung von Kundenpräferenzen für Produkte und Dienstleistungen. In: Baier D, Brusch M (Hrsg) Conjoint-analyse - Methoden-Anwendungen-Praxisbeispiele. Springer, Berlin, S 3-18

Balderjahn I, Hedergott D, Peyer M (2009) Choice-Based Conjointanalyse. In: Baier D, Brusch M (Hrsg) Conjoint-analyse - Methoden-Anwendungen-Praxisbeispiele. Springer, Berlin, S 129-146

Bauer C, Lang D, von Zedlitz H (2019) Die digitale Transformation erfolgreich monetarisieren. (5):24-28. Bank, Köln

Bélanger F, Crossler RE (2011) Privacy in the digital age: a review of information privacy research in information systems. MISQ 35(5):1017-1042

Beresford AR, Kübler D, Preibusch S (2012) Unwillingness to pay for privacy: a field experiment. Econ Lett 117(1):25-27

Berger SV, Gensler S (2007) Online banking costumers: insights from Germany. J Internet Bank Commer 12(1):1-6

Bichler A, Trommsdorff V (2009) Präferenzmodelle bei der Conjointanalyse. In: Baier D, Brusch M (Hrsg) Conjoint-analyse - Methoden-Anwendungen-Praxisbeispiele. Springer, Berlin, S 59-72

Bitkom (2014) Alternativen zur Hausbank. https://www.bitkom.org/Presse/Presseinformation/Alternativenzur-Hausbank.html;. Zugegriffen: 10. März 2020

Buck C, Stadler F, Suckau F, Eymann T (2017) Nutzer präferieren den Schutz ihrer Daten. HMD 54(1):55-66

Carrascal JP, Riederer C, Erramilli V, Cherubini M, de Oliveira R (2013) Your browsing behavior for a big mac: economics of personal information online. In: Proceedings of the 22nd international conference on World Wide Web, S 189-200

Cofinpro (2021) Kontoeröffnung 2021: Das geht noch besser

Cohen SH (1997) Perfect Union. Mark Res 9(1):12-17

Culnan MJ, Armstrong PK (1999) Information privacy concerns, procedural fairness, and impersonal trust: an empirical investigation. Organ Sci 10(1):104-115

Deutsche Bundesbank (2019) Zahlungsverkehrs- und Wertpapierabwicklungsstatistik in Deutschland 2014-2018. Deutsche Bundesbank, Frankfurt a. M.

Dinev T, Hart P (2006a) An extended privacy calculus model for E-commerce transactions. Inf Syst Res 17(1):61-80

Dorfleitner G, Hornuf L, Schmitt M, Weber M (2016) FinTech-Markt in Deutschland, Abschlussbericht des Bundesfinanzministeriums, S 1-98

EY (2014) Winning through customer experience. EY Global Consumer Banking Survey

EY (2021) Digital Banking 2020 - Verbraucherumfrage Januar 2021

FAZ (2020) Mehr und höhere Gebühren auf Girokonten. https://www.faz.net/aktuell/finanzen/finanztestmehr-und-hoehere-gebuehren-auf-girokonten-16908742.html;. Zugegriffen: 30. Sept. 2020

Häcker J, Bekelaer F (2019) Daten sind das Öl der Zukunft. Bank 4:50-54

Hann I-H, Hui K-L, Lee S-YT, Png IPL (2007) Overcoming information privacy concerns: an information processing theory approach. J Manag Inf Syst 24(2):13-42

Hansen M-H, Malz M (2015) Kundenzufriedenheit als Erfolgsmotor im Retailbanking. In: Seidel M, Liebetrau A (Hrsg) Banking \& Innovation 2015, S 121-128

Huberman BA, Adar E, Fine LR (2005) Valuating privacy. IEEE Secur Priv 3(5):22-25

Jentzsch N, Harasser A, Preibusch S (2012) Monetising privacy-an economic model of the pricing of personal information. Report der European Union Agency for Cybersecurity (ENISA)

Kara A, Kaynak E, Kucukemiroglu O (1994) Credit card development strategies for the youth market: the use of conjoint analysis. Int J Bank Mark 12(6):30-36

Rolfes B, Bauersfeld T, Grabbe B (2005) Präferenzorientierte Gestaltung von Girokontomodellen. Forschungsbericht des European Center for financial services (ecfs) 
Schoeman FD (1984) Privacy: philosophical dimensions of the literature. In: Schoeman FD (Hrsg) Privacy: philosophical dimensions of the literature: an anthology. Cambridge University Press, New York

Smith HJ, Dinev T, Xu H (2011) Information privacy research: an interdisciplinary review. MISQ 35(4):989-1015

Strategy\& (2020) European open banking: only slightly ajar-Strategy\& payments and open banking survey September 2020

Tsai JY, Egelman S, Cranor L, Acquisti A (2011) The effect of Online privacy information on purchasing behavior: an experimental study. Inf Syst Res 22(2):254-268

Verma R, Iqbal Z, Plaschka G (2004) Understanding customer choices in E-financial services. Calif Manage Rev 46(4):43-67

Warren SD, Brandeis LD (1890) The right to privacy. Harv Law Rev 4(5):193-220

Weiber R, Mühlhaus D (2009) Auswahl von Eigenschaften und Ausprägungen bei der Conjointanalyse. In: Baier D, Brusch M (Hrsg) Conjoint-analyse - Methoden-Anwendungen-Praxisbeispiele. Springer, Berlin, S 43-58

Zinkhan FC, Zinkhan GM (1990) Using conjoint analysis to design financial services. Int J Bank Mark $8(1): 31-34$

\section{Weiterführende Literatur}

Allenby GM, Arora N, Ginter JL (1995) Incorporating prior knowledge into the analysis of conjoint studies. J Mark Res 32(2):152-162

Green PE, Srinivasan V (1978) Conjoint analysis in consumer research: Issues and outlook. J Consumer Res 5(2):103-123

Myers JH, Alpert MI (1997) Determinant buying attitudes: meaning and measurement. Mark Manag $6(2): 50-56$

Netzer O, Toubia O, Bradlow ET, Dahan E, Evgeniou T, Feinberg FM, Feit EM, Hui SK, Johnson J, Liechty JC, Orlin JB, Rao VR (2008) Beyond conjoint analysis: advances in preference measurement. Mark Lett 19(3+4):337-354

Verlegh PWJ, Schifferstein WNJ, Wittink DR (2002) Range and number-of-levels effects in derived and stated measures of attribute importance. Mark Lett 13(1):41-52 